\title{
JOINT PREVENTIVE REPLACEMENT IN AN OPERATIONAL PLANNING PHASE
}

\author{
Rommert Dekker, Erasmus University Rotterdm, NL \\ Ralph Wildeman, Erasmus University Rotterdam, NL
}

In this paper we address the problem of combining preventive replacements in a multi-component maintenance system in an operational planning phase. Given an infinite or a finite time horizon, decisions concerning replacement of components have to be made at discrete time epochs within this horizon. We present a heuristic procedure that specifies an action for a given epoch, based on the current state of the system and on an expected behaviour of the system in the near future.

Firstly, for each component an expected initial replacement moment is determined, according to a control limit that has been obtained for each component separately, using a Markov decision model. These initial moments induce a finite planning horizon that starts at the current epoch, and in which the moments may be shifted so as to allow joint replacement, which saves set-up costs. However, these shifts usually imply a deviation from the initial plan, which may involve penalty costs. The objective is to find an optimal grouping of the replacements planned within the current planning horizon. The combination of components jointly replaced at the current epoch, corresponds to the action to be chosen at this epoch. This is repeated for each epoch. Thus we apply a 'rolling horizon' within the given infinite or finite time horizon. With numerical examples and a comparison with the optimal strategy, both for an infinite and a finite time horizon, we aim to illustrate and to justify our approach. 